\title{
Circadian rhythms of locomotor activity in captive eastern rock sengi
}

\author{
I. van der Merwe ${ }^{1}$, M.K. Oosthuizen ${ }^{1}$, N.C. Bennett ${ }^{1,3}$ \& C.T. Chimimba ${ }^{1,2}$
}

\author{
${ }^{1}$ Mammal Research Institute (MRI), Department of Zoology and Entomology, University of Pretoria, Private Bag \\ X20, Hatfield, 0028 South Africa \\ ${ }^{2}$ DST-NRF Centre of Excellence for Invasion Biology (CIB), Department of Zoology \& Entomology, University of \\ Pretoria, Private Bag X20, Hatfield, 0028 South Africa \\ ${ }^{3}$ Department of Zoology, King Saud University, Riyadh 11451, Saudi Arabia
}

Correspondence: Christian T. Chimimba, Mammal Research Institute (MRI) and DST-NRF Centre of Excellence for Invasion Biology (CIB), Department of Zoology and Entomology, University of Pretoria, Private Bag X20, Hatfield, 0028 South Africa.

Email: ctchimimba@zoology.up.ac.za

Page heading: Circadian activity in the sengi

\begin{abstract}
We investigated locomotor activity rhythms in the little-studied wild-caught eastern rock sengi (Elephantulus myurus) from Goro Game Reserve, Limpopo Province, South Africa. To assess whether locomotor activity is endogenously entrained by the light-dark cycle, animals $(n=13)$ were subjected to three different light cycle regimes: a $12 \mathrm{~h}$ light/12 $\mathrm{h}$ darkness (LD) cycle, a total darkness (DD) cycle, and an inverse of the LD cycle (DL). Ten animals exhibited strong light entrainment under LD1 with the total percentage of activity during the light phase $(56.5 \% \pm$ $11.9 \%)$ significantly higher than during the dark phase $(43.5 \% \pm 11.9 \%)$. Eleven animals
\end{abstract}


expressed distinct endogenous free-running rhythms under DD (mean $\tau=23.6 \mathrm{~h} \pm 0.6 \mathrm{~h}$; range: $22.9 \mathrm{~h}-24.5 \mathrm{~h}$ ), with significant inter-individual variation. Under DL, the total percentage of activity was approximately equal during the light $(50.4 \% \pm 7.8 \%)$ and dark phase $(49.6 \% \pm$ $7.8 \%$ ). Elephantulus myurus was on average active $25 \%$ of the $24-\mathrm{h}$ day with a nocturnal-diurnal ratio of 0.8 under LD1 and exhibited locomotor activity under controlled conditions similar to that of closely related species in the wild. In $62 \%$ of the animals, activity was highest around dawn, lowest during the afternoon and intermittently expressed throughout the night. Little quantitative data are available on the daily locomotor activity rhythms of sengis particularly in response to the light-dark cycle. This study provides valuable quantitative data on locomotor activity rhythms in E. myurus.

Key words: circadian rhythm, locomotor activity, light-dark cycle, Elephantulus myurus

\section{Introduction}

Numerous properties of the physical environment fluctuate rhythmically with time. Despite the existence of non-photic cues (Hut, Mrosovsky \& Daan, 1999; Laemle \& Ottenweller, 1999), the light-dark cycle remains the most predictive zeitgeber and hence the primary synchronizer of daily physiological- or behavioural rhythms, i.e. circadian rhythms (Pittendrigh

\& Minis, 1964; Skene et al., 1999; Quintero, Kuhlman \& McMahon, 2003). Without the input of external time cues, such as under constant dark conditions, the circadian rhythm continues to express itself at a free-running period $(\tau)$ of close to $24 \mathrm{~h}$ (Aschoff, 1981; Czeisler et al., 1999; Refinetti, 2006). Such a free-running rhythm provides evidence for an internal biological clocklike mechanism, which in mammals is positioned in the suprachiasmatic nucleus (SCN; Stephan 
\& Zucker, 1972; Okamura, Yamaguchi \& Yagita, 2002; Froy, 2007). Consequently, most living eukaryotes have activity rhythms that display a particular daily pattern when entrained to the light-dark cycle.

The sengi family (Macroscelididae) consists of two subfamilies, the giant sengis (Rhynchocyoninae) and the soft-furred sengis (Macroscelidinae), of which only the latter are found in the southern African subregion. Of the soft-furred species, the genus Elephantulus primarily inhabits arid or semi-arid environments; with the eastern rock sengi (Elephantulus myurus) largely restricted to rocky kopjes and boulder fields (Rathbun, 1979). Sengis primarily feed on invertebrates and are cursorial (Skinner \& Chimimba, 2005).

Giant sengis are reported as predominantly diurnal, with most of their active hours spent foraging, and there also appears to be an increase in their activity during the middle of the day (Rathbun, 1979, 2009). In contrast, there is great variation in the activity patterns described for the soft-furred species. Additionally, the literature provides conflicting descriptions of daily activity patterns and it would appear that soft-furred sengis are less strictly diurnal than has been originally reported (Rathbun, 1979; Woodall, Woodall \& Bodero, 1989; Mzilikazi \& Lovegrove, 2005). There are also only a few studies (Rathbun, 1979; Woodall et al., 1989; Ribble \& Perrin, 2005) that have presented quantitative data with regard to sengi activity rather than basing it upon either $a d$ hoc trapping regimes or sporadic field sightings, as appears to be the case in many literature reports. However, despite the quantitative data presented by Woodall et al. (1989), it was intimated that more is needed to understand daily activity patterns of sengis. This was primarily due to either the small sample size, or the presence of too many variables, during the course of their studies. Rathbun (2009) has furthermore reported that unlike giant sengis, several soft-furred species do not hide in nests, but rather utilize sheltering points amongst rocks and 
thickets from which they are easily disturbed, which may potentially further bias day-time anecdotal observations of their activity in the field.

All soft-furred species are most likely polycyclic and frequently display reduced activity during the middle of the night and the middle of the day with activity primarily peaking around sunrise and sunset (Rathbun, 1979; Woodall et al., 1989; Ribble \& Perrin, 2005). Some species such as the round-eared sengi (Macroscelides proboscideus), are reported as primarily nocturnal (Roxburgh \& Perrin, 1994), whilst others such as E. myurus are described as primarily diurnal (Woodal et al., 1989). Sengis exhibit various different activity behaviours such as grooming, foraging, inter-specific interactions and trail-cleaning (Rathbun, 1979; Ribble \& Perrin, 2005), which may affect the temporal distribution of activity differently between species. It has furthermore been suggested that the ratio of nocturnal to diurnal activity of sengis may be related to the amount of cover within the environment, which in turn is related to environmental temperature and the amount of exposure to predators (Woodall et al., 1989).

To date there has been no study to directly test for the internal control of locomotor activity by means of photic entrainment and thus determine a free-running period of locomotor activity for any sengi. Additionally, when investigating the locomotor activity rhythm of a species, it is naturally important to consider the organism's endogenous response to one of the most potent environmental entraining agents, the light-dark cycle. The aims of the study were thus to: 1) determine whether captive E. myurus possess a locomotory activity circadian pattern; and if so, 2) ascertain if it can be entrained to a controlled light-dark cycle; and 3) calculate the period of the free-running rhythm $(\tau)$ under constant dark conditions. 


\section{Materials and methods}

\section{Animal capture and maintenance}

Thirteen E. myurus (nine males; $49.74 \pm 2.71 \mathrm{~g}$, and four females; $51.92 \pm 4.41 \mathrm{~g}$ ), were collected at Goro Game Reserve in the Soutpansberg region, Limpopo Province, South Africa (between $22^{\circ} 58^{\prime} \mathrm{S}, 22^{\circ} 57^{\prime} \mathrm{S} ; 2^{\circ} 25^{\prime} \mathrm{E}, 2^{\circ} 24^{\prime} \mathrm{E}$ ) between the end of June and the beginning of July 2010. Specimens were collected using Sherman live traps (H. B. Sherman Traps, Inc. Tallahassee, Florida, U.S.A.) baited with a mixture of peanut butter, oats and fish oil. The species was positively identified using molecular tools and blasting against all known sengis in Genbank (Medger, 2010).

The experimental animals were maintained in a light-controlled room ( \pm 500 lux $)$ with a constant ambient temperature of $25^{\circ} \mathrm{C}\left( \pm 1^{\circ} \mathrm{C}\right)$ and $60 \%$ relative humidity $(\mathrm{RH})$. The animals were housed separately in polyurethane cages $(60 \times 30 \times 30 \mathrm{~cm})$ and provided with a layer of wood shavings and a small plastic container as shelter. The shelters were completely open at the one end which allowed light to always reach the animals (during the light phases). Each container had a wire mesh top to prevent the animals from escaping. All animals were given $a d$ libitum access to food and water which was provided daily at random times throughout the experiment in order to prevent entrainment by the feeding routine. The animals were fed a combination of Pronutro (Bokomo Foods, South Africa), canned dog food, carrots and apples. The sengis were housed and maintained according to the guidelines of the American Society of Mammalogists (Gannon, Sikes \& Animal Care and Use Committee, 2007) and the approval of the Animal Ethics Committee of the University of Pretoria, Pretoria, South Africa under its Ethics Clearance Number EC028-07. 


\section{Experimental procedure and activity recording}

The animals were acclimated for a period of two weeks on a 12L:12D light cycle, with the light phase starting at $0700 \mathrm{~h}$ and the dark phase starting at $1900 \mathrm{~h}$. After the acclimation period, all animals were exposed to four consecutive light cycle regimes (LD1, DD, LD2 and DL) each lasting between 18 and 20 days. During the LD1 light cycle, animals were subjected to the exact same lighting regime as during the acclimation period, in order to record any patterns of activity entrainment to the light-dark cycle. The animals were then subsequently subjected to a period of total darkness (DD) to assess the potential presence of an endogenous rhythm of locomotor activity and also to determine the free-running period. The next light cycle (LD2) again comprised of a light $(0700 \mathrm{~h}-1900 \mathrm{~h})$ phase and a dark phase $(1900 \mathrm{~h}-0700 \mathrm{~h})$, each lasting 12 $\mathrm{h}$, to re-entrain all animals before exposing them to the final light cycle (DL), which was an inverse of the LD light cycle. The aim of the DL light cycle was to investigate the effect of a drastic change in the light-dark cycle on the activity patterns of E. myurus.

An infra-red captor (Quest PIR internal passive infrared detector; Elite Security Products (ESP), Electronic Lines, U.K.) was fitted centrally onto the wire mesh cover of each cage and connected to a computer outside the experimental room. The infra-red captors detected and recorded all movements made within the container, which was then taken as the locomotor activity of the sengis. The number of locomotory movements made during each minute was collectively recorded and one value was presented for every minute using the computer program VitalView (VitalView ${ }^{\mathrm{TM}}$, Minimitter Co., Sunriver, OR, U.S.A.; www.minimitter.com) and the data were down-loaded and expressed as double-plotted actograms of two consecutive days using the same software. Each actogram presented a visual layout of the magnitude and frequency of activity recorded throughout each day for all the light cycles. 


\section{Data analysis}

Circadian locomotor activity was examined in E. myurus irrespective of sex, age, reproductive status or any other interspecific differences. The extent to which each animal was active during either the light phase or the dark phase of each light cycle regime was expressed as the percentage of activity. It was calculated for each animal during each light cycle regime as: the sum of all of the active counts during either the light- or dark phases divided by the total active counts and multiplied by 100 . Due to a technical error, percentage of activity of animal number five during LD2 was excluded. The mean percentage of activity for all animals during each light cycle regime yielded a corresponding total percentage of activity value. It should be noted that the percentage of activity did not express how activity was distributed across the day, for this a visual interpretation of the actograms was needed. We also calculated the mean amount of time that the animals were active during the dark phase, the light phase and the whole day of LD1, LD2 and DL. All per-minute data points were first converted to either 0s (no activity detected during that minute) or 1s ("active minutes": any amount of activity detected during that minute) in order to rule out any variation in the magnitude of daily activity between individuals. It was calculated as: the total number of "active-minutes" during a light cycle regime, divided by the total number of minutes of a light cycle regime ( $24 \mathrm{~h}$ x $60 \mathrm{~min} \times$ duration of light cycle regime in days), multiplied by 100 . The total "active-minutes" of the dark phase was then also divided by that of the light phase in order to establish a nocturnal-diurnal ratio (N:D) for LD1.

The period of the endogenous rhythm under constant dark conditions (DD) was determined by means of the eye-fit method using the ActiView tau cursor line. All of the actograms were visually examined in order to describe and interpret basic locomotor activity patterns of E. myurus within and across different light cycle regimes. Means \pm standard 
deviations were given where applicable to express variation. Further statistical analyses were performed using Microsoft Excel ${ }^{\mathrm{TM}}$ and Statistica ${ }^{\mathrm{TM}}$ version 9.0 (StatSoft Inc. 2009). Analysis of variance (ANOVA) for repeated measures was used to test for significant differences in percentage of activity between light- and dark phases within and across light cycles (LD1, LD2 and DL), with the post hoc Fisher's Least Significant Difference (LSD) test being used where significant differences were detected. Student's $t$-test was used to test for significant differences in the free-running periods between individuals.

\section{Results}

The daily locomotor activity rhythms of ten E. myurus individuals entrained strongly to the LD1 light cycle, but were less distinct in the remaining three animals. In Fig. 1, the actogram of one sengi with a distinctly and typically entrained circadian locomotor activity rhythm is displayed in contrast to an actogram representative of a sengi with unclear entrainment. Throughout the following DD light cycle, the same strongly entrained individuals had activity rhythms with equally distinct patterns (Fig. 2) but these rhythms became disorderly once the animals were reexposed to the light-dark cycle (LD2). Entrainment was thus present but not as unequivocal as during the LD2 light cycle.

Elephantulus myurus was mostly diurnal; during LD1 and LD2 only three animals were more nocturnal than diurnal. Locomotor activity was expressed in both phases (light and dark) of the 24-h day in all animals and most animals had a distinguishable daily activity pattern. There was a significant difference in the percentage of activity between light and dark phases within and across light cycles (ANOVA: $F_{2,48}=6.86 ; n=13 ; P<0.05$ ). The animals exhibited significantly more locomotor activity during the light phase than during the dark phase in both 
the LD1 light cycle (light phase: $56.5 \% \pm 11.9 \%$; dark phase: $43.5 \% \pm 11.9 \%$; Fisher's LSD: d.f. $=13,48 ; n=13 ; P<0.05$ ) and LD2 light cycle (light phase: $55.5 \% \pm 9.4 \%$; dark phase: $44.5 \% \pm$ 9.4\%; Fisher's LSD: d.f. $=12,48 ; n=13 ; P<0.05$; Fig 3 ). The percentage of diurnal activity ranged between $77.8 \%$ and $29.1 \%$ during the LD1 light cycle, and between $73.6 \%$ and $39.7 \%$ during LD2 light cycles, whereas the percentage of nocturnal activity ranged between $10.9 \%$ and 22.2\% during LD1, and between $60.3 \%$ and $26.4 \%$ during LD2 (Fig. 4). Despite differences in the percentage of activity expressed between individuals, distinguishable patterns in the daily activity rhythm of E. myurus could be established from the actograms. In eight animals nocturnal activity was expressed intermittently throughout the dark phase and diurnal activity concentrated to approximately the first half of the light phase. Activity commonly started to increase at $0400 \mathrm{~h}$ and attained a major peak at dawn. This was followed by a gradual decrease in activity until midday which occasionally included a small activity peak. The animals were least active during the afternoon and presented an intermediate activity peak at dusk prior to exhibiting intermittent activity throughout the dark phase (Figs. 1 and 5). During LD1, only one animal (animal number 2) exhibited diurnal activity above $70 \%(70.87 \%)$ and only one animal (animal number 11 ) exhibited nocturnal activity above 70\% (70.87\%; Fig 2 and Fig 4). There was no significant difference in the total percentage of activity between the light and dark phases of the DL light cycle (light phase: $49.6 \% \pm 7.8 \%$; dark phase: $50.4 \% \pm 7.8 \%$; Fisher's LSD: d.f. $=13,48 ; n=13$; $P=0.84$; Fig. 3) with six animals being slightly more active during the dark phase. Reentrainment to the DL light cycle was noticeable in eight animals and took between four and six days (Fig. 6).

Comparisons between the light phases of the three light cycles (LD1, LD2 and DL) revealed a significant difference between LD1 and DL (Fisher's LSD: d.f. $=13,48 ; n=13 ; P<$ 
0.05 ) as well as between LD2 and DL (Fisher's LSD: d.f. $=13,48 ; \mathrm{n}=13 ; P<0.05$ ). Similarly, there was a significant difference between the percentage of activity during the dark phase of LD1 and DL (Fisher's LSD: d.f. $=13,48 ; n=13 ; P<0.05$ ) as well as between LD2 and DL (Fisher's LSD: d.f. $=13,48 ; n=13 ; P<0.05)$. Neither the light (Fisher's LSD: d.f. $=13,48 ; n=$ $13 ; P=0.75)$ nor the dark phases (Fisher's LSD: d.f. $=13,48 ; n=13 ; P=0.75$ ) of LD1 differed significantly from that of the LD2 light cycle.

After transforming the per-minute activity points into activity present (1) and activity absent (0) values for each minute, it was found that the animals were on average active $25.2 \%$ (LD1), 27.4\% (LD2) and 25.2\% (DL) of the 24-h day (Table 1). N:D ratios of $0.81,0.82$ and 0.95 were also obtained for these three light cycles, respectively (Table 1). When subjected to constant darkness (DD), the locomotor activity rhythm persisted and provided evidence for an internal time-keeping mechanism. Elephantulus myurus displayed circadian rhythmicity with a significant amount of inter-individual variation in $\tau$ ranging from $22.9 \mathrm{~h}$ to $24.5 \mathrm{~h}$, and had a mean $\tau$ of $23.6 \mathrm{~h} \pm 0.6 \mathrm{~h}$ (Student's t-test: $t$-value $=143.17$; d.f. $=10 ; n=11 ; P<0.05$ ). Six of the animals had distinct free-running periods close to but less than $24 \mathrm{~h}$, the one primarily diurnal animal had a $\tau$ of longer than $24 \mathrm{~h}(\tau=24.5 \mathrm{~h}$; Fig. 2), four animals displayed a $\tau$ equal to $24 \mathrm{~h}$ (Fig. 1), and for the remaining two animals, activity was too scattered to be determined.

\section{Discussion}

The daily rhythms in locomotor activity of an organism are crucial to its survival in the natural environment (DeCoursey et al., 1997; Kronfeld-Schor \& Dayan, 2003). Mammalian circadian rhythms of locomotor activity are foundationally coordinated by the light-dark cycle (Daan \& Aschoff, 1981; Benstaali et al., 2001; Refinetti, 2006) but may be masked by other non- 
photic environmental stimuli such as food or temperature (Aschoff, 1981; Kronfeld-Schor \& Dayan, 2008). An animal's activity profile is usually defined by the temporal phase (day/night/twilight) during which it is most active and field observations are often used to classify animals as nocturnal, diurnal or crepuscular. However, field observations are unable to state unequivocally whether a particular activity profile is as the result of light-entrainment and not just due to the entrainment by a non-photic cue that overwrites the endogenous lightentrained rhythm, i.e. masking (Kronfeld-Schor \& Dayan, 2008). Therefore, in the present study, we exposed eastern rock sengis to a series of light-cycle regimes in a controlled environment in which the presence or the absence of light was the only external time cue.

Soft-furred sengis have previously been described as exhibiting activity to varying extents during both the day and at night (Rathbun, 1979; Woodall et al., 1989; Roxburgh \& Perrin, 1994; Ribble \& Perrin, 2005), yet little is known about the entrainment of locomotor activity in sengis by means of the environmental light-dark cycle. In addition, Rathbun (1979), Woodall et al. (1989) and Mzilikazi \& Lovegrove (2005) concur that there are many conflicting statements within the literature with regard to locomotor activity patterns recorded for the softfurred sengis. Perhaps apart from reflecting differences in the descriptive measures used, these differences may indicate that the soft-furred sengis readily adapt to differences in the environment and that the endogenous control of their activity may be less robust than in other species (Roll et al., 2006).

In the current study, E. myurus clearly possessed a light-entrained circadian locomotor activity rhythm. Our results revealed that most individuals expressed activity throughout the 24-h day (during LD1). The majority of the entrained animals did however exert significantly more activity during the day than at night and only one animal was strongly nocturnal (above $70 \%$ ) 
and another strongly diurnal (above 70\%). Similar proportions of nocturnal and diurnal activity were obtained by Woodall et al. (1989); E. myurus had an N-D ratio of 0.9, comparable to the 0.8 that we obtained. Interestingly, it is relatively uncommon for small-sized rodents to be active both during the day and night. For example, Roll et al. (2006) reported only one family (Geomyidae) out of six rodent families as being both nocturnally as well as diurnally active. However, species that are adapted to an insectivorous diet, such as the true shrews (Soricidae), frequently exhibit activity during the day and night (Mann \& Stinson, 1957; Ingles, 1960; Richardson, 1973). Similarly, E. myurus characteristically feeds on invertebrates, and as such does not hoard its food but presents a polycyclic activity rhythm (Rathbun, 1979; Mzilikazi \& Lovegrove, 2005). Elephantulus myurus is also capable of escaping extremely cold conditions by entering torpor (Mzilikazi \& Lovegrove, 2005) and in the present study, allocated a mean of only $13 \%$ more of its activity to the light phase than to the dark phase. Elephantulus myurus thus not only exerts activity at times during which the environment may potentially be cold (i.e., at night), but also that activity during such times might be necessary to meet their daily energy requirements. It is most likely that E. myurus prefers to exhibit intermittent activity throughout the night rather than during the afternoon when they are usually observed either sleeping or resting as a means of avoiding the hottest part of the day (Rathbun, 1979; Woodall et al., 1989).

The percentage of activity only expressed light-phase and dark-phase activity proportionately to each animal's total activity. From the actograms it was observed that the entrained animals had distinct daily activity patterns across the temporal time axis. In $61 \%$ of the animals nocturnal activity was intermittently expressed throughout the dark phase whilst diurnal activity was continuously expressed during roughly the first half of the light phase. The activity patterns of many sengi species, such as the four-toed sengi (Petrodromus tetradactylus), round- 
eared sengi (Macroscelides proboscideus), short-nosed sengi (E. brachyrhynchus), rufous sengi (E. rufescens), and E. myurus, tend to peak around twilight (Rathbun, 1979; Woodall et al., 1989). Similar to the preliminary findings of Woodall et al. (1989), most E. myurus individuals displayed one major activity peak at dawn and only in some cases a second, and considerably smaller, activity peak at dusk.

Furthermore, sengis take part in many different activity behaviours such as grooming, foraging, inter-specific interactions and trail-cleaning (Rathbun, 1979; Ribble \& Perrin, 2005). Animals housed in captivity are obviously unable to perform many of these activity behaviours and are thus expected to be slightly less active than they would in the wild. Our results revealed that E. myurus was on average only active approximately $25 \%$ of the 24 -h day, half of that estimated for the closely related E. rufescens (Rathbun, 1979). Interestingly, the daily activity peaks and times of least activity of E. myurus were still very similar to those of E. rufescens and P. tetradactylus observed under natural conditions (Rathbun, 1979), which demonstrates the adaptive value of an endogenously-controlled activity rhythm synchronized by the environmental light-dark cycle.

The period of the free-running rhythm of locomotor activity under constant lighting conditions has not been recorded for any sengi to date. The subsequent continuation of the activity rhythm under constant dark conditions (DD) provides evidence for the endogenous control of locomotor activity in E. myurus. Despite the wide range in $\tau$ between individuals within this species, our results still concurred with the tendency for a $\tau$ longer than $24 \mathrm{~h}$ to be associated with diurnal mammals and $\tau$ shorter than $24 \mathrm{~h}$ with nocturnal mammals (Aschoff, 1981; Fig. 2). The free-running activity periods of a vast number of animals, including invertebrates, reptiles, birds and mammals (primarily rodents), have been described and yielded a 
collective mean $\tau$ of $23.9 \mathrm{~h} \pm 0.7 \mathrm{~h}$ for 50 animal species (Refinetti, 2006) and is very close to the mean $\tau(23.6 \mathrm{~h} \pm 0.6 \mathrm{~h})$ obtained for E. myurus in this study. The value and the amount of variation in $\tau$ are usually species specific and may be influenced by various factors such as age, photoperiod, hormones, and light intensity (Pittendrigh \& Daan, 1974; Aschoff, 1981; Refinetti, 2006). Therefore, although it was not accounted for in this study, factors such as sex and age may have played a role in the large inter-individual variation obtained in this study. Nevertheless, perhaps these results suggest that many of the conflicting accounts of sengi activity are related to an inherent ability in this species to respond adaptively to photic stimuli.

In conclusion, when all other external time cues are absent, the daily locomotor activity rhythm of E. myurus shows clear entrainment to the light-dark cycle. This response is furthermore internally controlled as was revealed under constant dark conditions. Elephantulus myurus is generally active by day and by night but is significantly more active during the light phase. Interestingly, our study revealed similar daily locomotor activity rhythms to those of sengis in their natural environments and the internal control of locomotor activity in E. myurus most likely synchronizes its daily times of activity to favorable environmental conditions. Furthermore, the high inter-individual variation in $\tau$ reveals the adaptive potential of the internal rhythm to allow the animals to respond to unpredictable qualities of the environment. To date, very little quantitative information is available on this little-studied species regarding its daily patterns of locomotor activity. This study provides valuable insights into circadian locomotor activity rhythm of E. myurus.. 


\section{Acknowledgements}

The research was supported by a South African Research Chair of Mammal Behavioural Ecology and Physiology awarded to NCB. I. van der Merwe acknowledges a scholarship from the South African National Research Foundation (NRF). NCB acknowledges support of the Visiting professors program at King Saud University. The work was supported by a collecting permit from the Department of Nature Conservation in Limpopo Province. We thank Daniel Swanepoel and André Prins for assistance with fieldwork.

\section{References}

Aschoff, J. (1981). Freerunning and entrained circadian rhythms. In Handbook of behavioral neurobiology: Biological rhythms: 81-93. Aschoff, J. (Ed.). New York: Plenum Press.

Benstaali, C., Mailloux, A., Bogdan, A., Auzéby, A. \& Touitou, Y. (2001). Circadian rhythms of body temperature and motor activity in rodents. Their relationships with the light-dark cycle. Life Sci. 68, 2645-2656.

Czeisler, C.A., Duffy, J.F., Shanahan, T.L., Brown, E.N., Mitchell, J.F., Rimmer, D.W., Ronda, J.M., Silva, E.J., Allan, J.S., Emens, J.S., Dijk, D. \& Kronauer, R.E. (1999). Stability, precision, and near-24-hour period of the human circadian pacemaker. Science 284, 2177-2181.

Daan, S. \& Aschoff, J. (1981). The entrainment of circadian systems. In Handbook of behavioral neurobiology: Circadian clocks: 1-28. Takahashi, J. S., Turek, F. W. \& Moore, R. Y. (Eds). New York: Plenum Press. 
DeCoursey, P.J., Krulas, J.R., Mele, G. \& Holley, D.C. (1997). Circadian performance of suprachiasmatic nuclei (SCN)-lesioned antelope ground squirrels in a desert enclosure. Physiol. Behav. 62, 1099-1108.

Froy, O. (2007). The relationship between nutrition and circadian rhythms in mammals. Front. Neuroendocrinol. 28, 61-71.

Gannon, W.L., Sikes, R.S. \& The Animal Care and Use Committee of the American Society of Mammalogists (2007). Guidelines of the American Society of Mammalogists for the use of wild mammals in research. J. Mammal. 88, 809-823.

Hut, R.A., Mrosovsky, N. \& Daan, S. (1999). Nonphotic entrainment in a diurnal mammal, the European ground squirrel (Spermophilus citellus). J. Biol. Rhythms 14, 409-419.

Ingles, L. G. (1960). A quantitative study on the activity of the dusky shrew (Sorex vagrans obscurus). Ecology 41, 656-660.

Kronfeld-Schor, N. \& Dayan, T. (2003). Partitioning of time as an ecological resource. Annual Rev. Ecol. Evol. Syst. 34, 153-181.

Kronfeld-Schor, N. \& Dayan, T. (2008). Activity patterns of rodents: the physiological ecology of biological rhythms. Biol. Rhythm. Res. 39, 193-211.

Laemle, L.K. \& Ottenweller, J.E.. (1999). Nonphotic entrainment of activity and temperature rhythms in anophthalmic mice. Physiol. Behav. 66, 461-471.

Mann, P. M. \& Stinson, R. H. (1957). Activity of the short-tailed shrew. Can. J. Zool. 35, 171177.

Medger, K. (2010). The reproductive biology of two small southern African mammals, the spiny mouse, Acomys spinosissimus (Rodentia: Muridae) and the eastern rock elephant shrew, 
Elephantulus myurus (Macroscelidia: Macroscelididae). Unpubl. PhD thesis, University of Pretoria.

Mzilikazi, N. \& Lovegrove, B.G. (2005). Daily torpor during the active phase in free-ranging rock elephant shrews (Elephantulus myurus). J. Zool. (Lond.) 267, 103-111.

Okamura, H., Yamaguchi, S. \& Yagita, K. (2002). Molecular machinery of the circadian clock in mammals. Cell Tissue Res. 309, 47-56.

Pittendrigh, C. S. \& Minis, D. H. (1964). The entrainment of circadian oscillations by light and their role as photoperiodic clocks. Am. Nat. 98, 261-294.

Pittendrigh, C.S. \& Daan, S. (1974). Circadian oscillations in rodents: a systematic increase of their frequency with age. Science 186, 548-550.

Quintero, J.E., Kuhlman, S.J. \& McMahon, D.G. (2003). The biological clock nucleus: a multiphasic oscillator network regulated by light. J. Neurosci. 23, 8070-8076.

Rathbun, G.B. (1979). The social structure and ecology of elephant-shrews. Adv. Ethol. 20, 1-75.

Rathbun, G.B. (2009). Why is there discordant diversity in sengi (Mammalia: Afrotheria: Macroscelidea) taxonomy and ecology? Afr. J. Ecol. 47, 1-13.

Refinetti, R. (2006). Circadian physiology. Boca Raton: 2nd edn. CRC Press/Taylor \& Francis Group.

Ribble, D.O. \& Perrin, M.R. (2005). Social organization of the eastern rock elephant-shrew (Elephantulus myurus): the evidence for mate guarding. Belg. J. Zool. 135 (supplement), $167-173$.

Richardson, J. H. (1973). Locomotory and feeding activity of the shrews, Blarina brevicauda and Suncus murinus. Am. Midl. Nat. 90, 224-227. 
Roll, U., Dayan, T. \& Kronfeld-Schor, N. (2006). On the role of phylogeny in determining activity patterns of rodents. Evol. Ecol. 20, 479-490.

Roxburgh, L. \& Perrin, M.R. (1994). Temperature regulation and activity pattern of the roundeared elephant shrew Macroscelides proboscideus. J. Therm. Biol. 19, 13-20.

Skene, D.J., Lockley, S.W., Thapan, K. \& Arendt, J. (19990. Effects of light on human circadian rhythms. Repro. Nutr. Dev. 39, 295-304.

Skinner, J.D. \& Chimimba, C.T. (2005). The mammals of the southern African subregion. 3rd edn. Cambridge: Cambridge University Press.

Stephan, F.K. \& Zucker, I. (1972). Circadian rhythms in drinking behavior and locomotor activity of rats are eliminated by hypothalamic lesions. Proc. Natl. Acad. Sci. USA 69, 1583-1586.

Woodall, P.F., Woodall, L.B. \& Bodero, D.A.V. (1989). Daily activity patterns in captive elephant-shrews (Macroscelididae). Afr. J. Ecol. 27, 63-76. 
Table 1 Mean percentages of time per day spent active as well as nocturnal-diurnal (N:D) ratios obtained for the eastern rock sengi, Elephantulus myurus from South Africa subjected to three different light cycles (see Materials and methods section).

\begin{tabular}{llll}
\hline & \multicolumn{4}{l}{ Mean \% of time per day spent active $( \pm \mathrm{SD})$} \\
\cline { 2 - 4 } Light cycle & LD1 & LD2 & DL \\
\hline Light phase & $13.9 \pm 6.7$ & $15.1 \pm 5.9$ & $12.9 \pm 5.5$ \\
Dark phase & $11.2 \pm 5.3$ & $12.3 \pm 4.9$ & $12.3 \pm 6.1$ \\
Total & 25.1 & 27.4 & 25.2 \\
N:D & 0.8 & 0.8 & 1 \\
\hline
\end{tabular}
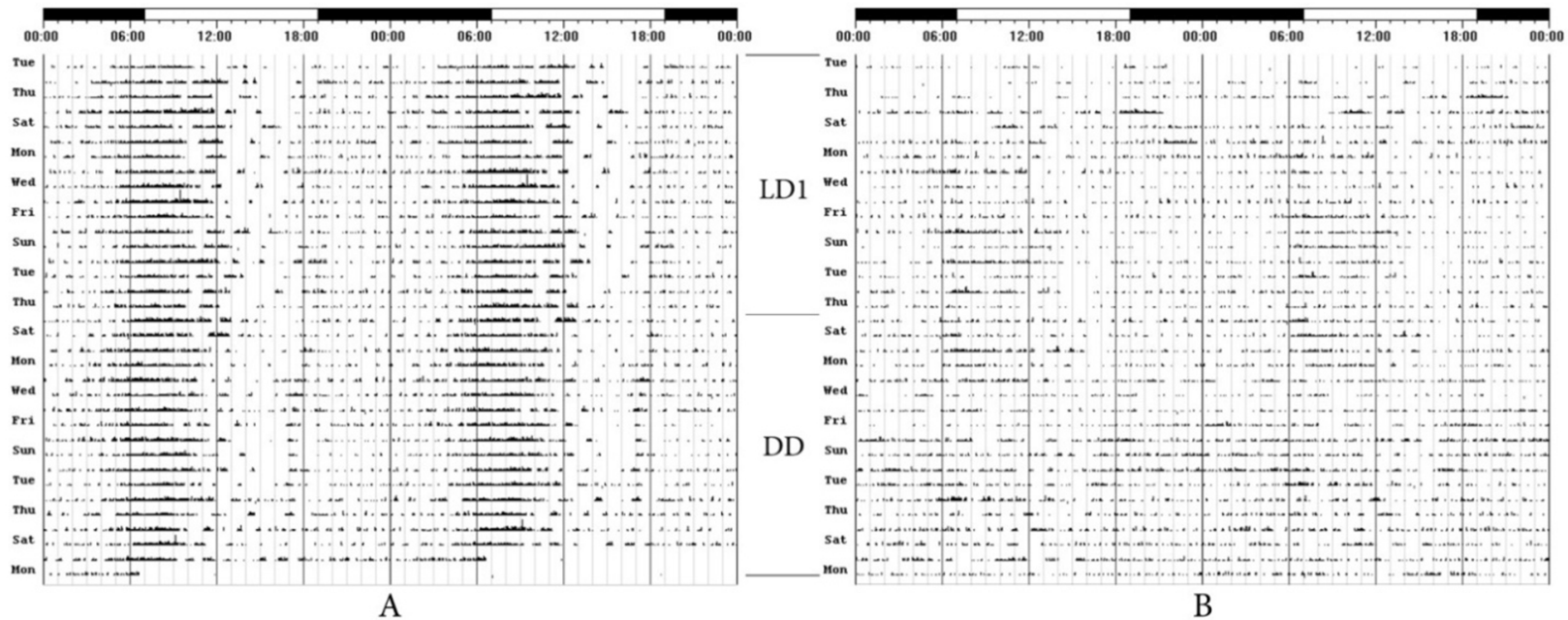

$\mathrm{B}$

Figure 1 Representative actograms of: A) an eastern rock sengi with a typical and distinctly entrained locomotor activity rhythm (animal number four) and a free-running period of $24 \mathrm{~h}$ under the DD cycle and B) a sengi with unclear entrainment of locomotor activity (animal number 12). The number of days is represented by the Y-axis with 18 days during the LD1 cycle and 19 days during the DD cycle. The solid and open bars at the top of each actogram represent the dark- and light phases of the LD1 cycle, respectively. During DD the animals were exposed to constant darkness. 


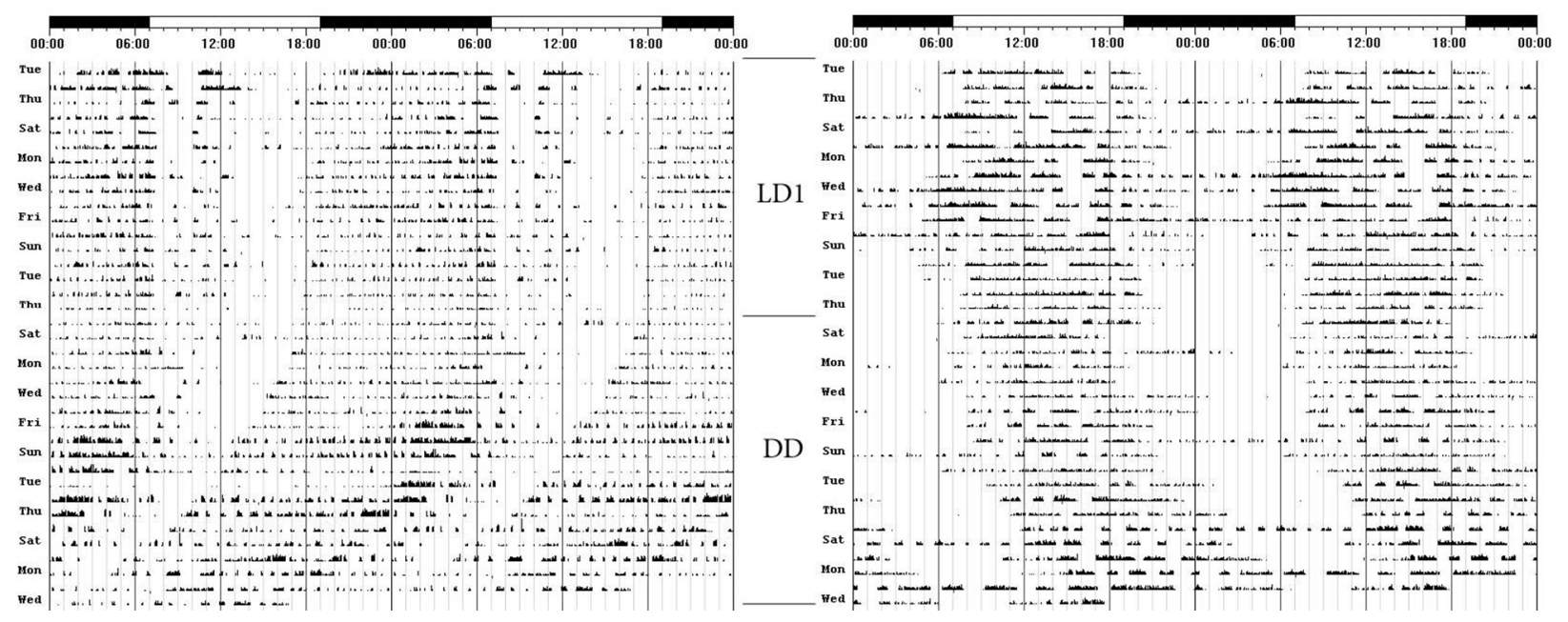

Figure 2 Actograms of the eastern rock sengi showing examples of: A) a free-running period close to but less than $24 \mathrm{~h}$ (animal number 11), and B) a free-running period of longer than $24 \mathrm{~h}$ (animal number 2). The number of days is represented by the $\mathrm{Y}$-axis with 18 days during the LD1 cycle and 19 days during the DD cycle. The solid and open bars at the top of each actogram represent the dark- and light phases, respectively. During DD the animals were exposed to constant darkness. 


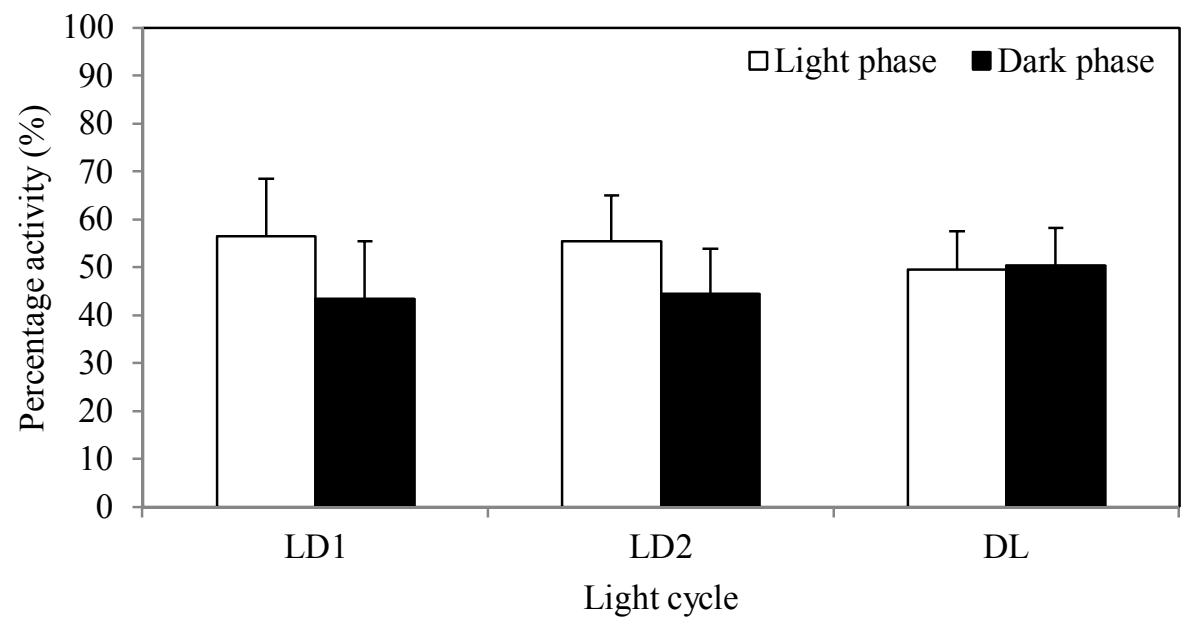

Figure 3 The total percentage of activity of the eastern rock sengi, Elephantulus myurus, obtained during the light and dark phases of the LD1, LD2 and DL light cycles (see Materials and methods section), respectively. 

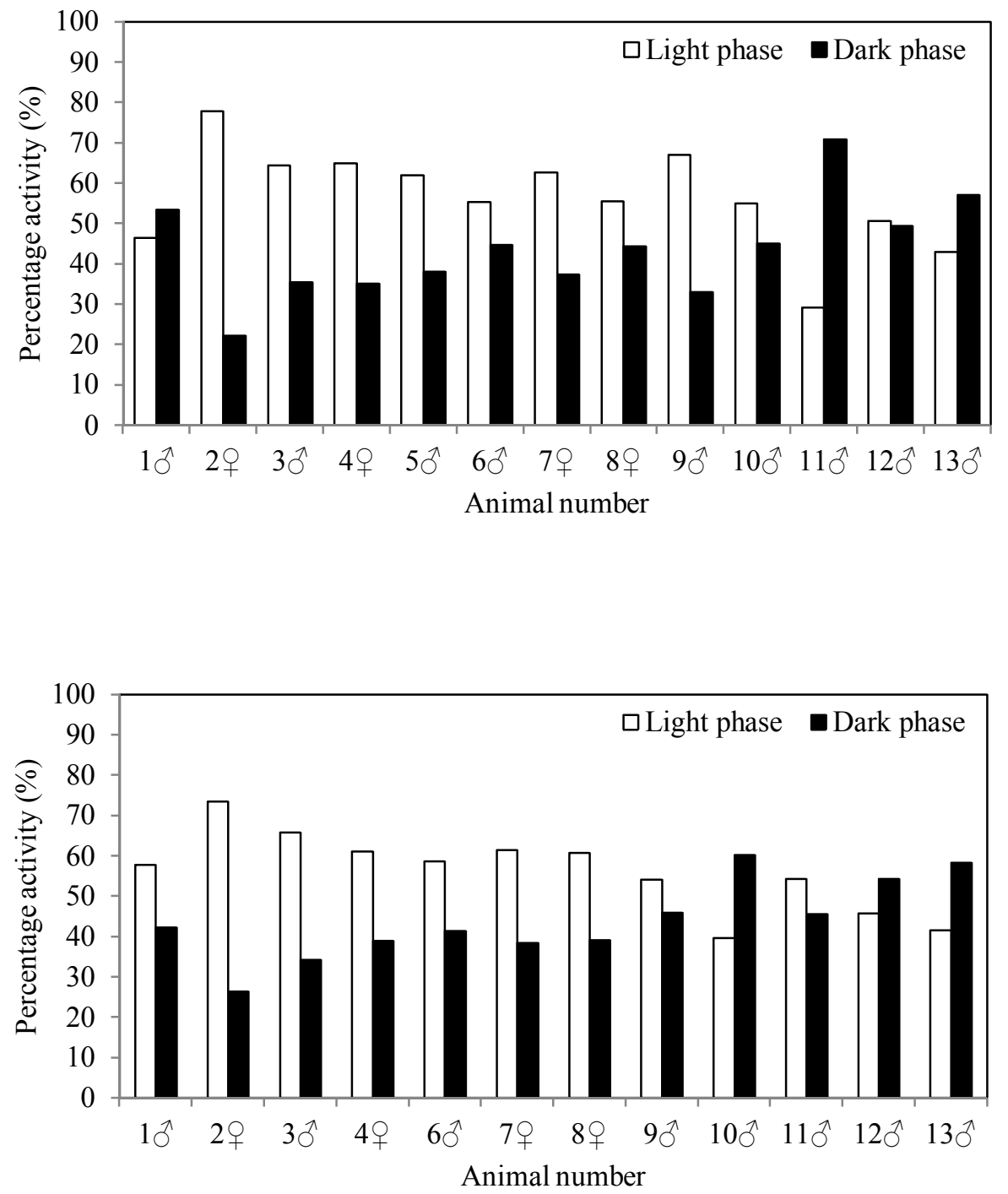

Figure 4 Individual percentages of activity for the eastern rock sengi, Elephantulus myurus, exhibited during the light phase and dark phase of the LD1 (top; 13 animals) and LD2 (bottom; 12 animals) light cycles (see Materials and methods section). 


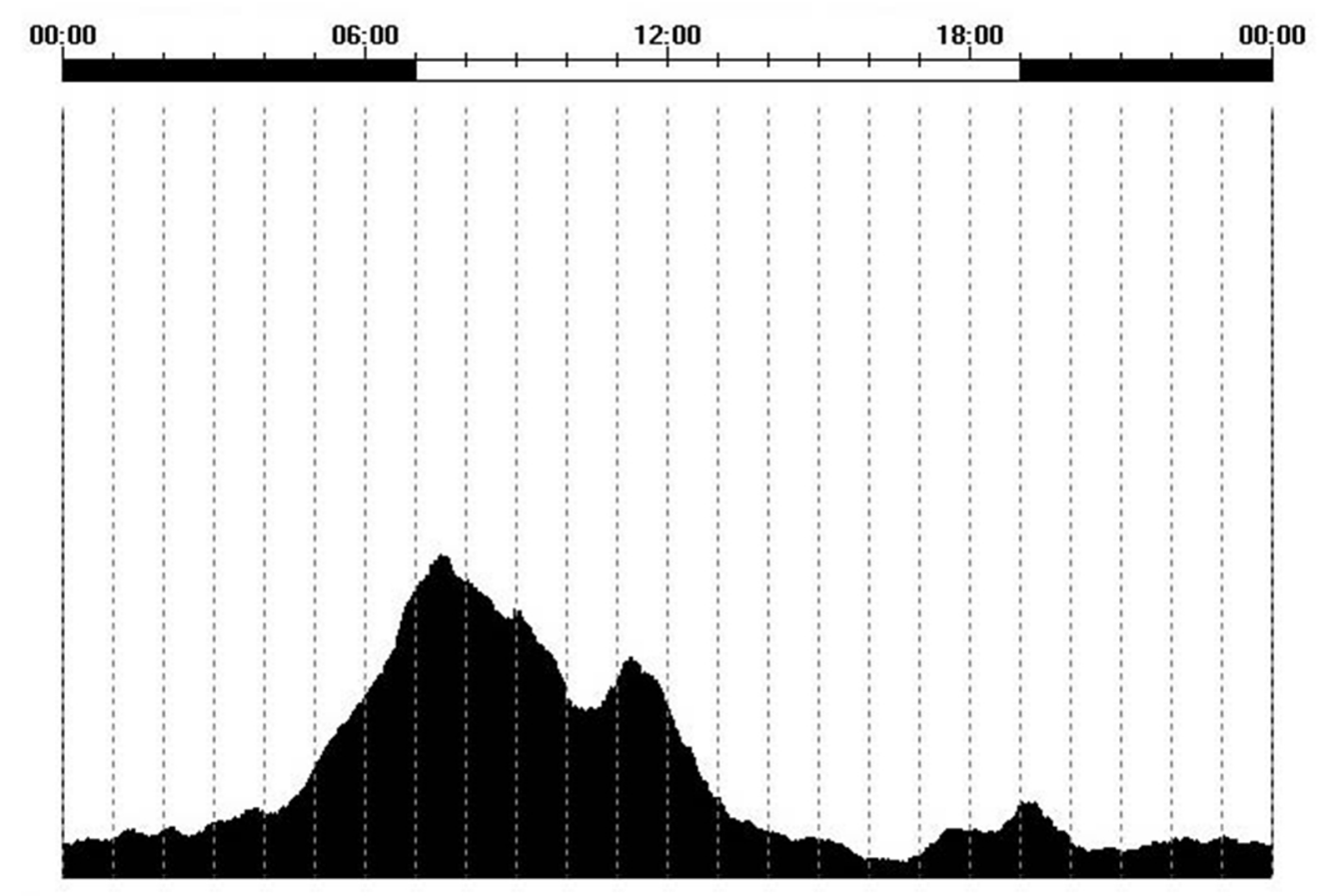

Figure 5 An example of an activity profile of the eastern rock sengi, for the LD1 light cycle (see Materials and methods section), illustrating a major activity peak at dawn and a minor activity peak at dusk. The solid and open bars at the top represent the dark and light phases, respectively. 


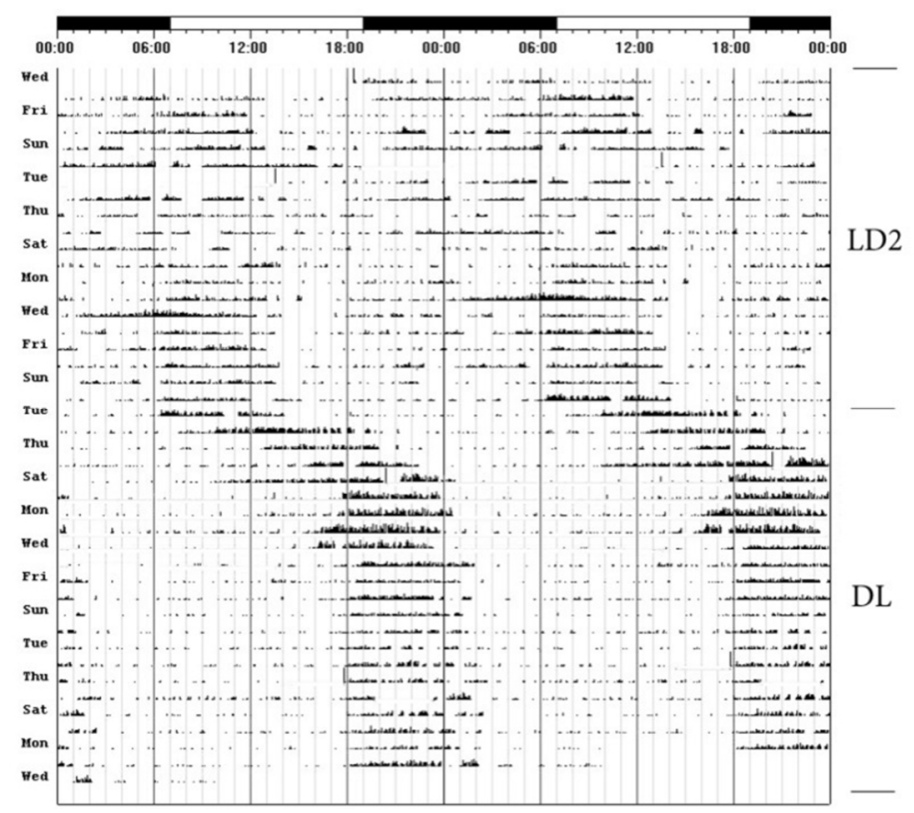

Figure 6 An actogram showing the re-entrainment of locomotor activity of the eastern rock sengi, to an inverse of the LD light cycle (animal number nine). The solid and open bars at the top of each actogram represent the light and dark phases, respectively and are inverted during the DL cycle. The Y-axis represents the number of days with 19 days during LD2 and 20 days during DL. 